zione allegorico-gestuale, in cui le espressioni corporee sono analizzate all'interno del contesto narrativo di riferimento, si passa ad un'analisi dettagliata dei gesti infernali ora decontestualizzati rispetto alla cornice narrativa, isolati e valutati in sé. L'analisi sistematica da' vita ad un'ampia e dettagliata casistica in cui la fenomenologia delle figure e dei movimenti fisici viene descritta mediante la distinzione tra le parti del corpo che originano un determinato movimento. Testa, volto, occhi, braccia, mani, gambe e piedi sono gli attori principali di un repertorio preverbale analizzato minuziosamente e posto in essere dai protagonisti del viaggio oltremondano: Dante, Virgilio, i condannati, i diavoli, i guardiani e le altre inquietanti presenze del regno dei morti. In totale i gesti dell'Inferno sono 319, la maggior parte di essi riguarda i peccatori. Ne risulta un'evidente relazione tra gestualità e peccato, soprattutto considerando che l'organo che in questo caso è il più presente nella produzione dei gesti sono le mani, che il peccato, appunto, commisero. Segue Dante, come maggior produttore di gestualità, ma l'organo più sollecitato è, nel caso del protagonista, quello della vista. Nel cammino iniziatico verso la visione finale della verità lo sguardo, allegoria della conoscenza, è il vero protagonista della mimica corporale dantesca, mentre la zona che si distingue per il tasso più alto di espressioni nonverbali è quella delle malebolge, il regno della frode. Lo studio dei gesti, conclude Violeta Díaz-Corralejo, può quindi fornire una visione distinta o più completa dei canti infernali mediante la decodificazione di un canale di trasmissione di senso alternativo a quello linguistico e che, interpretato in una prospettiva esegetico-allegorica, può, come lo studio delle similitudini, fornire importanti informazioni per una più completa, $\mathrm{o} a$ volte diversa e nuova, lectura del testo dantesco.

\section{Chiara Cappuccio}

\title{
Francesco FURLAN
}

\section{Studia albertiana. Lectures et lecteurs de L. B. Alberti}

Torino-Paris: Nino Aragno Editore - J. Vrin, 2003.

Alcuni anni di lavoro attorno alla figura e alla cultura di Alberti sono raccolti da F. in questo ampio volume, nel segno della definizione di un progetto globale di scelta dalla parte umanistica intesa a un sapere concreto, a una scienza non superflua né superficiale oltreché da subito schierata - e sono le pagine prime dell'opera in argomento - contro i valori esteriori (contro l'alchimia, ad es.) e pure in nome di un ideale di utilitas che è tanto dinamico quanto ancorato a fondamenti di certezza epistemologica e tout court scientifica. Lintuizione del dialogo come genere-contenitore meglio adatto alla discussione su questi ordini di faccende viene dunque considerata, e giustamen- te, da F. come luogo qualificante un intero, lucido versante del programma umanistico di Alberti, dove la storia tecnica dello strumento dialogico - un ibrido anche per gli antichi, Luciano in primis docente - viene fatta ascendere alle stesse difficoltà discretive denunciate da Platone in merito ai rapporti del genere col criterio fondamentale della mimesi: allo specchio dunque di una riflessione lunga diversi secoli che col principio medesimo di credibilità storica aveva dovuto fare $\mathrm{i}$ conti $a b$ ovo, solo a pensare, come fa F., a due dialoghi lucianeschi ben presenti ad Alberti quali la Calumnia o Quomodo historia sit conscribenda. Fu allora l'istinto di verosimiglianza del dialogo d'area cice- 
roniana a inscrivere nel genere quei princìpi di ricerca collettiva della verità, "examen en commun des opinions et des connaissances", che nell'articolato capitolo Méthodes, formes et contexte, p. 63 s., si presentano sotto forme plurime, di assoluto controllo entro il progetto ciceroniano di sintesi tra filosofia e vita passato in eredità - una volta lasciate indietro le limitazioni di stampo platonizzante alla forte tradizione tre-quattrocentesca della dialogistica umanistica. L'A. mostra con estrema e raffinata chiarezza argomentativa i modi grazie ai quali il valore antico della conversatio (si recuperano per questo persino i dittaggi di Bartolomeo da S. Concordio di monastica attinenza culturale) è preponderante in Alberti sino ai tempi del De iciarchia ma specialmente sui fondamenti di un'esigenza di rinascita "volgare» dello strumento dialogico, scalata entro due insiemi produttivi: da un lato i quattro libri De familia, Sofrona, i Profugiorum ab aerumna libri III, la Cena familiaris e appunto i tre libri del De iciarchia, dall'altro Deifira e i tre libri del Theogenius. È dopo aver proceduto a definire le differenti stagioni (e ragioni) compositive dei due nuclei di sviluppo concettuale albertiano in merito al canone volgare del dialogo che F. si volge a studiare attentamente le prerogative, forme e modi di funzionamento, tipologia e connotati strutturali o ricorrenti del genere dialogico proprio dei testi estremi albertiani, che «doivent être étudiés dans une même perspective, [...] parce qu'ils constituent un segment à part et fort spécifique de l'oeuvre d'Alberti: soit qu'ils se réclament explicitement d'une même conception du dialogue comme d'un ragionare domestico e familiare régi avant tout par une exigence de mimêsis et de réalisme; soit qu'ils se caractérisent à l'inverse par une négation déliberée de cette exigence et que, fuyant la réalité, ils se présentent comme des dialogues "rêvés" ou oniriques s'engageant entre des interlocuteurs immatériels, à l'extrême limite du monde humain, voire précisément dans U-topie - une utopie à plusieurs égards "nocturne"» (p. 96).

In effetti, configurandosi il De familia come «une tentative consciente de lecture de la réalité humaine à partir de la famille», l'alternativa utopica e onirica di Theogenius o di alcune delle Intercoenales fa da sfondo di contrasto esattamente ai princìi «realistici» di definizione dello spazio-tempo urbano-familiare che reggono alcuni dei più rilevanti dialoghi volgari (eccezion fatta, ovviamente, per i summenzionati Deifira e Theogenius), in nome e sotto l'egida di un ideale di concretezza del mondo domestico che nelle pagine dell'A. di espande ad alcuni esiti ben individuati dei Profugia e persino del De iciarchia; con quell' incremento di verosimiglianza, descrittiva e argomentativa, che Alberti ha scelto di appoggiare sul trovato della presenza costante del personaggio-autore (p. 113 s.). Così la strutturazione autenticamente dialogica dell'opera albertiana viene percorsa in profondo nel capitolo quarto della prima parte (Dialogue et vérité dans le De familia), dove la costruzione riflessiva dei personaggi-interlocutori viene piegata dallo scrittore, sul cammino della conoscenza, verso l'obiettivo sommo di un sapere empirico per pruova e non per coniettura: alla fine «l'ensemble des techniques ou des méthodes du dialogue antique se retrouvent [...] dans le De familia, où leur combinaison permet aux conversation d'avancer en prenant toujours en compte une pluralité d'experiences et de points de vue ainsi que des modes de connaissance virtuellement opposés».

La seconda parte del volume riguarda più in specifico i modi della diffusione discontinua, non lineare dell'opera di Alberti, tra le censure d'area fiorentina e la ridotta seppur talvolta ammirata circolazione francese: si eleggeranno gli esempi di Ecatonfilea e di Deifira quali testi-prova più fortunati in questo contesto in forza della loro partecipazione - 
almeno parziale - alla tradizione novellistica, tanto da consentire all'A. di ottenere un profilo tutto sommato coerente dei lettori tardi di quei testi (come Mirabeau) - ed è il capitolo Traductions et adaptations à la veille de la Révolution. Ecatonfilea, Deifira et leur lecteurs, p. 173 s. L'addizione di Simiae al gruppo dei testi dell'Alberti meno "fortunato", la conferma del Naufragus e la sostanziale cancellazione dell'Istorietta (viziata peraltro dalla storia di quel vistoso falso del Bonucci che nel ms. Cerchi 16 dell'Archivio di Stato di Firenze), sino all'importante restituzione di testo + figura del $17^{\circ}$ ex ludis rerum mathematicarum sono alcune delle oramai note quanto decisive acquisizioni di F. nel corso del suo lungo tirocinio di filologo e lettore di testi; laddove con la terza parte dell'opera i differenti modi di una lettura tematica centrata sui concetti di famiglia e di donna sullo sfondo del quadro borghese della ricordanza portano sì a rilevare un certo qual distacco albertiano dalle radicate concezioni ecclesiastiche in argomento, ancora giusta «la lutte entre un principe d'individualisme, qui est également culte personnel d'une ratio morale, et un besoin de reconnaissance sociale, d'utilité, d'élan vers les autres, d'une action plus concrète», ma anche consentono di leggere la susseguente, albertianamente misogina condanna della figura della donna nella chiave dello schema del disordine umano-irra- zionale da sempre combattuto dall'umanista. Lo stesso recupero interpretativo della Dissuasio Valerii, operato da F. nel capitolo De Gauthier Map à Leon Battista Alberti. Sur la misogynie comme remedium amoris et cura sui, riesce a sigillare a dovere un poderoso insieme di ricerche come questo, integrandone liminarmente il significato con le parole che riassumono la coerenza e del libro e delle sue singole sezioni nei confronti della "stagione del dialogo" consegnataci da uno dei giacimenti più vasti della cultura albertiana: «La transposition en volgare ou le remaniement de la Dissuasio Valerii qu'entend constituer et constitue de fait la Risposta albertienne se développe donc selon deux directions principales qui peuvent être définies comme une laïcisation radicale du texte d'une part et, de l'autre, comme une réecriture qui semble marquée en profondeur par l'émergence de valeurs et d'idéaux humanistes - pour ne pas dire albertiens - tels que l'autonomie de la culture, le besoin de la recherche historique ou érudite, la nécessité des arts, de l'investigation philosophique». Giacimento che F. ha avuto il merito di studiare nel tempo così nel suo complesso come nelle singolari esecuzioni storiche, con una lucidità di sguardo costantemente nutrita dalla ratio philologica che si può dire in tutto ammirevole.

Marcello Ciccuto

\section{Giacomo LEOPARDI}

\section{Cants,}

traducció i notes de Narcís Comadira, edició bilingüe, Barcelona: Edicions 62-Empúries, 2004.

Siamo particolarmente contenti di presentare ai nostri lettori questa straordinaria versione dei Canti di Leopardi a cura del poeta catalano Narcís Comadira. L’opera maggiore del poeta recanatese è finalmente approdata al catalano grazie alla versione di uno dei più valenti traduttori e poeti contemporanei. Sembra sia arrivata l'ora che una delle voci più intense della poesia universale di tutti i tempi, ma anche una delle più incomprese dalla critica in generale, giunga comprensibile ai 